\title{
Selective electrochemical sensing of human serum albumin by semi-covalent molecular imprinting
}

\author{
Maciej Cieplak, ${ }^{a, *}$ Katarzyna Szwabinska, ${ }^{a, b}$ Marta Sosnowska, ${ }^{a, c}$ Chandra Bikram KC, ${ }^{c}$ \\ Pawel Borowicz, ${ }^{a, d}$ Krzysztof Noworyta ${ }^{a}$ Francis D'Souza, ${ }^{c, *}$ and Wlodzimierz Kutner ${ }^{a, e, *}$ \\ ${ }^{a}$ Institute of Physical Chemistry, Polish Academy of Sciences (IPC PAS), Kasprzaka 44/52, 01-224 Warsaw, Poland \\ ${ }^{b}$ Institute of Chemistry and Technical Electrochemistry, Poznan University of Technology, Berdychowo 4, 60-965 \\ Poznan, Poland \\ ${ }^{c}$ Department of Chemistry, University of North Texas, 1155 Union Circle, \#305070, Denton, TX 76203-5017, USA \\ ${ }^{d}$ Institute of Electron Technology, Al. Lotnikow 32/46, 02-668 Warsaw, Poland \\ ${ }^{e}$ Faculty of Mathematics and Natural Sciences, School of Science, Cardinal Stefan Wyszynski University in Warsaw, \\ Wóycickiego 1/3, 01-815 Warsaw, Poland
}

\begin{abstract}
We devised and prepared a conducting molecularly imprinted polymer (MIP) for human serum albumin (HSA) determination using semi-covalent imprinting. The bis(2,2'-bithien-5yl)methane units constituted the MIP backbone. This MIP was deposited as a thin film on an Au electrode by oxidative potentiodynamic electropolymerization to fabricate an electrochemical chemosensor. The HSA template imprinting, and then its releasing from the MIP was confirmed by the differential pulse voltammetry (DPV), electrochemical impedance spectroscopy (EIS), XPS, and PM-IRRAS measurements as well as by AFM imaging. Semi-covalent imprinting provided a very well defined locations of recognition sites in the MIP molecular cavities. These sites populated the imprinted cavities or the MIP surface only. The DPV and EIS response of the MIP film coated electrode to the HSA analyte was linear in the range of 0.8 to 20 and 4 to 80 $\mu \mathrm{g} / \mathrm{mL} \mathrm{HSA}$, respectively, with the limit of detection of 16.6 and $800 \mathrm{ng} / \mathrm{mL}$, respectively. The impressively high imprinting factor reached, exceeding 20, strongly confirmed that semicovalent imprinting resulted in formation of a large number of very well defined molecular cavities with high affinity to the HSA molecules. The MIP selectivity against low-(molecular weight) interferences, common for physiological fluids, such as blood and urea, was very high. There was no response to the presence of these interferences at concentrations encountered in the samples analyzed. Moreover, the chemosensor selectivity to the myoglobin and cytochrome $c$ interferences was excellent while that to lysozyme was slightly lower but still high. The
\end{abstract}


chemosensor was useful for determination of abnormal HSA concentration in a control blood serum.

\section{Keywords}

Human serum albumin, semi-covalent imprinting, molecularly imprinted polymer (MIP), electrochemical chemosensor, thiophene conducting polymer, differential pulse voltammetry, electrochemical impedance spectroscopy, blood serum albumin.

\section{Corresponding author}

\section{Maciej Cieplak}

e-mail: mcieplak@ichf.edu.pl,

Tel: +48223433188

Fax: +48223433333 


\section{Introduction}

Human serum albumin (HSA) is an essential component of the protein fraction in human blood plasma. HSA constitutes nearly half a blood serum protein (Peters 1995). Its molecular weight and dimensions are, $M_{\mathrm{w}}=66.4 \mathrm{kDa}$ and $7.5 \times 6.5 \times 4.0 \mathrm{~nm}^{3}$, respectively (Erickson 2009). HSA is mainly responsible for transport of several important substances and xenobiotics in human body including hormones, fatty acids, and drugs (Curry et al. 1998). Moreover, it maintains oncotic pressure and prevents photodegradation of folic acid. HSA concentration in blood plasma is typically 35 to $50 \mathrm{mg} / \mathrm{mL}$. Abnormal HSA concentration can serve as an indicator of a coronary heart disease (Danesh et al. 1998) and multiple myeloma (Greipp et al. 2005). A low level of HSA in plasma (hypoalbuminemia) indicates liver failure, cirrhosis, and chronic hepatitis (Murch et al. 1996). A moderate HSA concentration (30 to $300 \mu \mathrm{g} / \mathrm{mL}$ ) in urea is indicative of microalbuminuria and higher concentrations of albuminuria, both caused by kidney damage, most often resulting from diabetes and hypertension (Hoogenberg et al. 1993). Therefore, the HSA determination is so much clinically important.

Colorimetry is now the most commonly used method of HSA determination. It relies on Bromocresol Green binding (Rodkey 1965). However, it suffers from serious disadvantages. Most importantly, the HSA concentration is often overestimated with this method because equilibrium of the HSA reaction with this dye is not reached and, therefore, the analytical response is time dependent. Apparently, this response is rising fast all the time (Snozek et al. 2007; Webster et al. 1974). Moreover, Bromocresol Green may react with other proteins too (Keay and Doxey 1984). Typically, the linear dynamic concentration range extends from 100 to $300 \mu \mathrm{g} / \mathrm{mL}$ HSA (Doumas et al. 1997). Furthermore, fluorescence spectroscopy, often used for determination of HSA, is generally inadequate with respect to selectivity and stability (Hawe et al. 2008; Kessler et al. 1997; Matulis et al. 1999; Sun et al. 2012; Suzuki and Yokoyama 2005; Volkova et al. 2011). This is because the HSA emission can easily be interfered by the fluorescence of other proteins in biological samples. Only quite recently, the first, based on the Pittsburgh Green II dye, fluorescence protocol of HSA determination was developed affording the time independent response to HSA. This response was linear in the range of 2 to $10 \mu \mathrm{g} / \mathrm{mL}$ HSA (Smith et al. 2014). Moreover, a label-free, based on silicon nitride $\left(\mathrm{Si}_{3} \mathrm{~N}_{4}\right)$, impedimetric HSA immunosensor was fabricated (Caballero et al. 2012). This sensor was highly selective with respect to other proteins and its response was linear in the range of $10^{-13}$ to $10^{-7} \mathrm{M}$ HSA. 
However, it revealed disadvantages typical of immunosensors. That is, its fabrication was rather complicated and expensive. Besides, stability of anti-HSA antibodies used was limited. Moreover, this immunosensor could operate as a disposable device only because its sensitivity dropped by as much as $40 \%$ when HSA was extracted from its recognizing film after the first use. Therefore, we heiren used molecular imprinting for HSA determination.

Molecularly imprinted polymers (MIPs), synthesized with the use of macromolecular templates $\left(M_{\mathrm{w}}>1500 \mathrm{Da}\right)$, have received significant attention for nearly last two decades. The task of devising the protein recognizing MIPs has already been challenged by developing different imprinting procedures. These are commonly categorized as the bulk, particle, surface, and epitope imprinting (Bergmann and Peppas 2008; Bossi et al. 2007; Kryscio and Peppas 2012; Li et al. 2014; Verheyen et al. 2011; Yang et al. 2012). Despite of a vast research in this field, only limited progress has been made so far. The most promising seems to be molecular imprinting of an epitope, i.e., devising an MIP with only a small part of a peptide used as the template (Rachkov and Minoura 2001). However, this promising approach has its limitations. That is, the fragment chosen for imprinting should be specific for the target protein only and, moreover, it should readily be accessible for binding, e.g., it should be located on surface of the target protein molecule. Furthermore, this epitope should easily be available at a reasonable cost.

Some attempts to fabricate MIP chemosensors selective with respect to HSA have already been undertaken. In one, an Au-coated quartz crystal resonator (Au-QCR) was spin coated with a film of the HSA imprinted polyacrylamide using 3-dimethylaminopropyl methacrylamide (DMAPMA) as the functional monomer (Lin et al. 2004). This resonator was then used to determine HSA by piezoelectric microgravimetry (PM) with a quartz crystal microbalance (QCM). However, the linear dynamic concentration range was rather narrow $(60-160 \mu \mathrm{g} / \mathrm{mL})$ and linearity of the response was low. In another attempt, a molecularly imprinted with HSA silicon sol-gel system was deposited on an Au-QCR (Zhang et al. 2006). Then, HSA was simultaneously determined using PM and electrochemical impedance spectroscopy (EIS). This chemosensor was very selective with respect to several peptides and the response to HSA was linear in the range of $10^{-9}$ do $10^{-6} \mathrm{~g} / \mathrm{mL}$. Moreover, a selective to HSA acrylic MIP bearing an organic dye ( $O$-acryl-L-4-hydroxyproline amide of dansylethylendiamin) inside the imprinted cavity was preapered (Inoue et al. 2013). The linear dynamic concentration range of the 
fluorescence response of this MIP chemosensor was 0.5 to $5 \mu \mathrm{M}$ HSA. Selectivity of this chemosensor with respect to the lysozyme, avidin, and chymotrypsin interferences was high. However, it was low to bovine serum albumin (BSA). The fluorescence of the polymer was quenched only because of HSA binding in the cavities and not because of non-specific HSA adsorption on the MIP surface. For this hypothesis verification, the same MIP was deposited on surface of an SPR chip. In this case, both the imprinting factor and selectivity dramatically dropped.

Imprinting of peptides is quite challenging mainly because of their large size and conformation liable to experimental conditions. In case of non-covalent imprinting (Sellergren et al. 1988), it is very difficult to estimate which and how many binding sites are accessible for the recognition on the template molecule. For that, selection of a proper molar ratio of the functional monomers to the peptide monomers in the solution for polymerization seems to be crucial. That is, too high concentration of the functional monomer would lead to the appearance of the recognizing functionalities unbound to the template molecules and randomly distributed in the MIP. This randomization would result in a low imprinting factor and low selectivity on the one hand, however, on the other too low ratio would lead to a lower number of recognition sites in the molecular cavities and, hence, lower affinity to the peptide analyte. Therefore, optimization of the composition of the pre-polymerization solution for the non-covalent imprinting is time and labor consuming. To circumvent these difficulty, we herein introduced a semi-covalent approach to protein imprinting. This imprinting involves covalent binding of recognition moieties of functional monomers with binding functional groups of the template (Sellergren and Andersson 1990). Then, after polymerization, the template must be removed by cleavage of these covalent bonds. But in contrast to a typical covalent imprinting (Wulff and Sarhan 1972), the analyte binds to the imprinted cavity only in a non-covalent way in this semicovalent approach, e.g., with the hydrogen bonds and/or via electrostatic, van der Waals, and hydrophobic interactions. By definition, this imprinting assures that all functional monomers incorporated in the MIP matrix are bound to the template. Until now, the semi-covalent imprinting was not used for protein templates. There are some examples of MIP fabrication where proteins were covalently immobilized on the substrate surface (Moreira et al. 2013a; Moreira et al. 2013b; Moreira et al. 2013c; Tretjakov et al. 2013). Moreover, cytochrome $c$ was labeled with a cleavable functional monomer bearing a disulfide bond and terminal maleimide 
moiety capable of binding cys-17 located on the cytochrome $c$ surface (Suga et al. 2013). After MIP deposition, the disulfide bond was cleaved, and then the resulting thiol moiety was used for post-imprinted labeling of the molecular cavity with a fluorescent organic dye. Apparently, molecularly imprinted cavities were formed in a typical non-covalent way in all these examples.

In the present paper, we describe a novel approach to imprinting of macromolecular compounds, represented by the HSA protein. For that, HSA was semi-covalently imprinted in a thiophene based conducting MIP. Until now, semi-covalent imprinting was successfully used only for imprinring small-molecule compounds (Sellergren 2001). Here, our MIP was deposited as a thin film on an $\mathrm{Au}$ electrode surface by electropolymerization under potentiodynamic conditions to serve as the recognition unit of an electrochemical chemosensor for selective HSA determination.

\section{Materials, instrumentation, and procedures}

\subsection{Materials and Reagents}

All chemicals, solvents, and peptides were from Sigma-Aldrich except of 2,2'-bithiophene-5carboxylic acid (Enamine, Ltd.) as well as $N$-(3-dimethylaminepropyl)- $N^{\prime}$-ethylcarbodiimide hydrochloride (EDC) and 1-hydroxy-7-azabenzotriazole (HOAt) (TCI N.V.). The NORTROL control serum (with HSA concentration 38 to $48 \mathrm{mg} / \mathrm{mL}$ ) was from Thermo Fischer Scientific.

\subsection{Instrumentation}

A VSP potentiostat/galvanostat computerized electrochemistry system of Bio-Logic, SAS, controlled by the CE-Lab V10.37 software of the same manufacturer, was used for the CV, DPV, and EIS measurements. A one- or two-mL three-electrode one-compartment V-shaped glass electrochemical minicell was used. A 2-mm in diameter Au disk sealed in a soft glass tubing, an $\mathrm{Ag} \mid \mathrm{AgCl}$, and a coiled $\mathrm{Pt}$ wire was used as the working, pseudo-reference, and counter electrode, respectively. The infrared spectra of thin films were recorded using polarization-modulation infrared reflection-absorption spectroscopy (PM-IRRAS) with a Vertex 80v Fourier transform infrared (FTIR) spectrophotometer of Bruker, equipped with a PMA50 PM-IRRAS module. The nitrogen-cooled MCT (Hg-Cd-Te) detector served to obtain a reasonably high signal-to-noise ratio. All IR spectra were analyzed with the OPUS 6.5 software of Bruker. 
The MIP films were imaged by AFM (in the Tapping ${ }^{\mathrm{TM}}$ mode) with a MultiMode 8 AFM microscope of Bruker under control of a Nanoscope V controller. The films for imaging were deposited on the Au-film coated glass slides.

The XPS spectra were recorded on a PHI 5000 VersaProbe (ULVAC-PHI) scanning ESCA microprobe using monochromatic $\mathrm{Al} \mathrm{K \alpha}$ radiation $(h v=1486.6 \mathrm{eV})$. The Casa XPS software was used to evaluate the XPS data. Background was subtracted using the Shirley method and peaks were fitted with the (Gaussian-Lorentzian)-shaped profiles. The binding energy $(B E)$ scale was referenced to the $\mathrm{C}$ 1s peak with $B E=284.6 \mathrm{eV}$.

The UV-vis spectra were recorded with 0.1-nm resolution using a UV-2501 spectrophotometer of Shimadzu.

\subsection{Procedures}

Syntheses of the $p$-bis(2,2'-bithien-5-yl)methylalanine cross-linking monomer and 5,5',5'methanetriyltris $\left(2,2^{\prime}\right.$-bithiophene) functional monomer were described elsewhere (Huynh et al. 2013a; Huynh et al. 2013d).

\subsubsection{Derivatizing of human serum albumin with functional monomers}

HSA (15 mg, $0.23 \mu \mathrm{mol})$ was suspended in a mixed solvent solution of toluene $(0.5 \mathrm{~mL})$ and chloroform (0.3 mL). Next, 2,2'-bithiophene-5-carboxylic acid 1 (11 mg, $52 \mu \mathrm{mol})$, HOAt (11 $\mathrm{mg}, 80 \mu \mathrm{mol})$, EDC $(0.015 \mathrm{~mL}, 13 \mathrm{mg}, 68 \mu \mathrm{mol})$, and triethylamine $(0.025 \mathrm{~mL})$ were added. After 10-h stiring at room temperature, $p$-bis(2,2'-bithien-5-yl)methylalanine 2 (40 mg, 92 $\mu \mathrm{mol})$, additional portion of EDC $(0.015 \mathrm{~mL}, 13 \mathrm{mg}, 68 \mu \mathrm{mol})$, and triethylamine $(0.02 \mathrm{~mL})$ were added, and then the suspension stirred for another $10 \mathrm{~h}$. The labeled HSA was extracted from the reaction mixture with the phosphate buffer saline, PBS, $(2 \times 0.5 \mathrm{~mL}, \mathrm{pH}=7.4)$ solution. Then, the HSA peptide was purified by liquid chromatography on a Sephadex column. Volume of the collected product fraction was $5 \mathrm{~mL}$ (labeled HSA concentration of $\sim 3 \mathrm{mg} / \mathrm{mL}$ ). The success of the derivatized HSA synthesis was proven by both the UV-vis and IR spectroscopy measurements (Fig. S1, Supplementary material).

\subsubsection{Synthesis of the molecularly imprinted polymer (MIP-HSA) film}


The MIP-HSA film was prepared by oxidative electropolymerization under potentiodynamic conditions with 50 potential cycles over the potential range of 0 to $1.20 \mathrm{~V}$ at the potential scan rate of $50 \mathrm{mV} / \mathrm{s}$ (Fig S.2, Supplemental Data). For preparation of a solution for the electropolymerization, the solution of labeled HSA $(0.1 \mathrm{~mL})$ was added to the acetonitrile $(0.9$

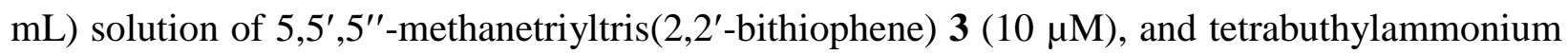
perchlorate $(100 \mathrm{mM})$. Before MIP-HSA film deposition, the electrode was immersed in the "piranha" solution for $10 \mathrm{~min}$, and then mirror finished with the alumina slurry $(0.05 \mu \mathrm{m})$. (Warning. The "piranha" solution is very dangerous if contacted with skin or eye.)

\subsubsection{Synthesis of the control non-imprinted polymer (NIP) film}

The NIP film was prepared by oxidative electropolymerization under the same conditions as those used for the MIP-HSA synthesis. The only difference was that free functional monomers were added to the polymerization mixture instead of the labeled HSA. The concentrations of functional monomers were adjusted to the concentrations that were used for MIP-HSA deposition assuming that HSA labelling was complete.

\section{Results and discussion}

\subsection{Labeling of human serum albumin with functional monomers}

For imprinting, we have chosen two different functional monomers, vis., one bearing the carboxylic group 1 and the other the amine group 2 (Frame A in Scheme 1). These monomers were then covalently bound to the appropriate functional groups on the HSA surface (Step $a$ in Scheme 1). For this labeling, a mixed solvent solution of toluene and chloroform was used. This solution afforded sufficient solubility of all the reactants and, moreover, it was much less destructive to the HSA peptide than other solvents were, including methanol and acetone (Klibanov 2001). 

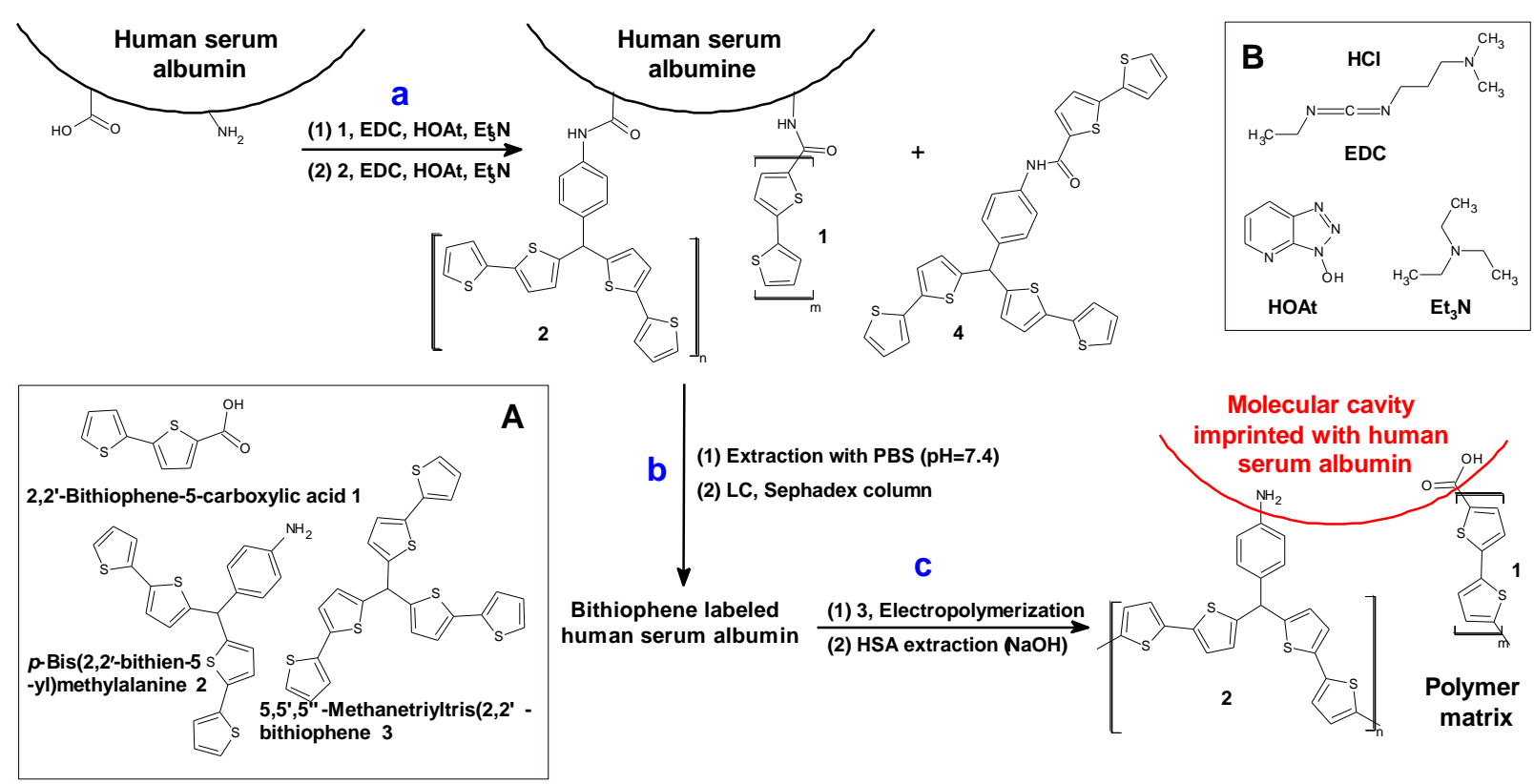

Scheme 1. ( $a$ and b) Derivatizing of human serum albumin with the bithiophene functional monomers 1 and $\mathbf{2}$, and (c) formation of the HSA imprinted molecular cavity in MIP. (Frame A) structural formulas of the 2,2'-bithiophene-

5-carboxylic acid 1, p-bis(2,2'-bithien-5-yl)methylalanine 2 functional monomers and the 5,5',5'methanetriyltris(2,2'-bithiophene) $\mathbf{3}$ cross-linking monomer as well as (frame B) structural formulas of EDC - N-(3dimethylaminepropyl)- $N^{\prime}$-ethylcarbodiimide hydrochloride, HOAt - 1-hydroxy-7-azabenzotriazole, and $\mathrm{Et}_{3} \mathrm{~N}$ triethylamine.

Our procedure of MIP preparation allowed using a large excess of functional monomers with respect to the number of binding sites on the HSA surface. Therefore, all functional groups available on surface of the HSA template molecule were bound. The excess of the functional monomers and the side product 4 were then removed by LC. Moreover, this procedure allowed using carboxylic and amine functional monomers simultaneously. Under other conditions (e.g., in a non-covalent imprinting approach), these monomers would interact with each other.

The success of HSA derivatizing was proven by both the UV-vis and IR spectroscopy measurements (Fig. S1, Supplemental Data). That is, new UV absorption bands at 220, 280, and $330 \mathrm{~nm}$ for the HSA solution, which appeared after labeling (spectrum 2 in Fig. S1a, Supplemental Data), evidenced this labeling. Moreover, new absorption bands corresponding to the presence of functional monomers are seen in the PM-IRRAS spectrum for the derivatized HSA (curve 2 in Fig. S1b, Supplemental Data). Particularly, the absorption bands characteristic of the amide bonds (especially, Amide I, II, and A) significantly increased. This increase proved 
that additional amide bonds were introduced to the HSA molecules in the course of the derivatizing.

\subsection{Preparation of the polymer molecularly imprinted with human serum albumin (MIP-} HSA)

The labeled HSA was used for preparation and deposition on the Au electrode of the MIP-HSA film. For that, we adopted our procedure that was successful for imprinting of small molecules (Huynh et al. 2013a; Huynh et al. 2013c; Pietrzyk et al. 2010a; Pietrzyk et al. 2010b). Then, the template was removed from the film by immersing the MIP-HSA coated electrode in $30 \% \mathrm{NaOH}$ for $45 \mathrm{~min}$ at $40^{\circ} \mathrm{C}$ until the DPV and EIS response of the chemosensor was constant. The DPV curves and EIS spectra of the chemosensor before and after electropolymerization as well as after template extraction are shown in Fig. 1.

[Here Fig. 1]

After template removal, the DPV curves and EIS spectra were very similar to those for the bare electrode. This behavior might result from emptying molecularly imprinted cavities, thus facilitating diffusion of the redox probe trough the polymer to the electrode surface for charge exchange.

Initially, we tested another MIP-HSA deposition procedure (Supplemental Data). However, this procedure was abandoned because the resulting MIP-HSA film was mechanically unstable.

\subsection{Characterization of the polymer molecularly imprinted with human serum albumin}

For characterization, the MIP-HSA film was deposited on an Au film coated glass slide. However, the deposition current was high and, therefore, a thin layer of gold was oxidized. To avoid this undesired effect, the solution for electropolymerization was diluted ten times.

The PM-IRRAS spectrum, recorded after deposition of the MIP-HSA film, showed a strong broad absorption band in the range of 1800 to $1000 \mathrm{~cm}^{-1}$ (spectrum 1 in Fig. S3, Supplemental Data), assigned to the HSA present in the film. Unfortunately, it was impossible to assign any bond vibration in this range because of signals strongly overlapped. After HSA extraction, this broad absorption band disappeared (spectrum 2 in Fig. S3, Supplemental Data). 
Moreover, signals characteristic of the peptide bonds (Amide I-III, and A) were absent. This absence proved a complete removal of the HSA from the MIP-HSA matrix.

The XPS spectrum recorded after deposition of the MIP-HSA film on the Au electrode showed a strong N1s signal of nitrogen $(B E=399.7 \mathrm{eV})$ and no evidence of the presence of sulfur (Table S1, Supplemental Data). This high nitrogen content and the sulfur absence may indicate that the MIP-HSA surface was completely coated by the HSA molecules, which were surface immobilized with the peptide bonds. After HSA extraction, the nitrogen content on the MIP surface significantly decreased (Table S2, Supplemental Data). Moreover, two sulfur signals appeared $(B E=163$ and $169 \mathrm{eV})$. But still, the nitrogen-to-sulfur atomic ratio was very high $\left(N_{\mathrm{N}}\right.$ : $N_{\mathrm{S}}=1.5: 1$ ). Apparently, all functional monomers in the MIP were precisely positioned (Scheme 1) and all of their functional groups were present on the MIP-HSA surface or on the surface of the imprinted cavities while the bithiophene moieties were deeply buried inside the MIP matrix. Moreover, for the NIP film, in which distribution of the monomers was random, the nitrogen-to-sulfur atomic ratio was much lower $\left(N_{\mathrm{N}}: N_{\mathrm{S}}=1: 4.8\right)$.

The MIP-HSA film was AFM imaged in order to unravel its morphological differences before and after HSA extraction (Fig. S4, Supplemental Data). Apparently, the MIP-HSA surface was quite flat and smooth consisting of 20 to $60-\mathrm{nm}$ grains of the bithiophene polymer (Fig. S4a, Supplemental Data). No smaller structures were seen on surface of these grains. Moreover, the visco-elastic properties changed only on edges of the grains (Fig. S4c and S4d, Supplemental Data). Besides, roughness of the film, i.e., the ratio of the 3-D area to that 2-D projected, only slightly decreased (from 16\% to 12\%) after extraction of HSA (Fig. S4b, Supplemental Data) suggesting that HSA was removed from the surface of the MIP grains. However, no information about cavities inside polymer grains was obtained. The AFM determined thickness of the MIP-HSA film was 75 to $100 \mathrm{~nm}$.

\subsection{Characterization of the MIP-HSA electrochemical sensor for determination of human serum albumin}

The DPV peak for the redox probe couple of $\mathrm{K}_{4} \mathrm{Fe}(\mathrm{CN})_{6} / \mathrm{K}_{3} \mathrm{Fe}(\mathrm{CN})_{6}$ at the MIP-HSA film coated Au disk electrode decreased faster the higher was the HSA concentration in the solution (Fig. S5,

Supplemental Data). Apparently, the redox probe diffusion through the MIP film was slowed down because of large HSA molecules entered the film. The drift in the DPV peak current 
resulted from at least two simultaneous processes. One involved slow diffusion of inorganic counter ions and/or a decreased level of polymer doping in a neutral solution. The other, presumably the most important, originated from slow HSA diffusion. Therefore, the peak measurement at $60 \mathrm{~min}$ after HSA addition to the test solution seemed to be the most appropriate time compromise between the measurement of reasonably stable DPV and EIS signals and the measurement ergonomics (Fig. S6, Supplemental Data).

Two electrochemical techniques were used for constructing the HSA calibration plots. That is, the DPV and EIS curves were simultaneously recorded at a carefully controlled time of 60 min after addition of HSA to the redox probe solution. In DPV, the HSA-extracted MIP film coated electrode linearly responded to the HSA concentration in solution in the range of 12 to $300 \mathrm{pM}$, i.e., 0.8 to $20 \mu \mathrm{g} / \mathrm{mL}$ (Fig. 2a) obeying the linear regression equation of $I_{60} / I_{0}=$

$0.67( \pm 0.01)-1.56 \times 10^{-4}\left( \pm 6 \times 10^{-5}\right) c_{\mathrm{HSA}}, \mathrm{pM}$ with the correlation coefficient of 0.9963 . The limit of HSA detection was 0.25 pM, i.e., $16.6 \mathrm{ng} / \mathrm{mL}$, (curve 2 in Fig. S5, Supplemental Data). The imprinting factor was impressively high, $\mathrm{IF}_{\mathrm{DPV}}=20.4$.

[Here Fig. 2]

In the EIS (Fig. 2b) measurements, the linear dynamic concentration range was 60 to 1200 $\mathrm{pM}$ (4 to $80 \mu \mathrm{g} / \mathrm{mL}$ ) satisfying the linear regression equation of $\Delta R_{\mathrm{ct}}, \Omega=654( \pm 66)+1.56( \pm 0.1)$ $c_{\mathrm{HSA}}, \mathrm{pM}$ with the correlation coefficient of 0.9803 . The limit of HSA detection was $12 \mathrm{pM}(0.8$ $\mu \mathrm{g} / \mathrm{mL}$ ) and the imprinting factor was even higher than that determined by DPV, i.e., it was $\mathrm{IF}_{\mathrm{EIS}}$ $=26.8$.

\subsection{Selectivity of the MIP-HSA chemosensor with respect to the low-(molecular weight) interferences}

Semi-covalent imprinting assures very well defined location of recognizing sites in an MIP. That is, these sites are encountered only in the imprinted molecular cavities or on the MIP surface. Therefore, selectivity of the MIP with respect to the low-(molecular weight) interferences, typical for body fluids including blood and urea, is expected to be very high. Those interferences, even if bound by the MIP, should not cause any polymer swelling and, moreover, they are too small to physically block the (several-nanometer)-size imprinted cavities. In the present study, the EIS responses of the MIP film coated electrode to those interferences, at maximum physiological concentrations, are compiled in Table 1. 


\section{[Here Table 1]}

Advantageously, the MIP-HSA film coated electrode did not respond to most tested low(molecular-weight) interferences including creatinine, urea, and uric acid. However, a relatively strong EIS signal was produced for glucose at its high concentration of $5.55 \mathrm{mM}(1 \mathrm{mg} / \mathrm{mL})$. Fortunately, a blood sample must be diluted a thousand times before the HSA detrmination. Therefore, the presence of glucose should have no effect on the detection signal. Blood cholesterol is usually bound to the transport proteins. Other possible interferences are present at much lower concentrations. Therefore, the devised MIP-HSA chemosensor meets all requirements of direct determination of HSA.

\subsection{Selectivity of the MIP-HSA chemosensor with respect to other peptides}

One of the most important aims of using molecular imprinting is to reach as high chemosensing selectivity with respect to analyte analogs as possible. To estimate this selectivity, the MIP-HSA chemosensor response to several different proteins was measured herein. Usually, concentrations of peptides are given in mass $(\mu \mathrm{g})$ per volume $(\mathrm{mL})$. But molecular weights of peptides may differ by several times. Therefore, molar concentrations are more reliable to express selectivity adequately (Fig. 3).

\section{[Here Fig. 3]}

Advantageously, the MIP-HSA chemosensor selectivity against myoglobin and cytochrome $c$ was excellent, i.e., the chemosensor was practically insensitive to these proteins (curves 3 and 4 , respectively, in Fig 3). Selectivity with respect to lysozyme was lower, equaling to 3.6, but still high (curve 2 in Fig 3). We can speculate that myoglobin and cytochrome c mostly consist of densely packed $\alpha$-helices that make their structures rigid. However, nearly half the lysozyme sequence consists of random coils that are flexible. Most likely, this is why lysozyme molecules fit, at least partially, to the shape and location of recognizing sites of the imprinted cavity.

\subsection{Determination of human serum albumin in artificial blood serum samples with the MIP-HSA chemosensor}

To prove that detectability of our chemosensor is sufficient for determination of abnormal concentrations of HSA in blood serum, we prepared samples of different concentrations of the 
NORTROL control serum in the absence and presence of known amounts of HSA. Then, the HSA concentrations in the samples were determined with the MIP-HSA chemosensor using EIS, and then recovery was determined (Table 2). The determined concentrations of HSA in samples were comparable to those prepared.

\section{[Here Table 2]}

\section{Conclusions}

We devised and fabricated an electrochemical chemosensor for selective determination of human serum albumin at the concentration level lower than that typical of human serum. We prepared this chemosensor by semi-covalent HSA molecular imprinting in a thiophene conducting polymer deposited as a thin film on an Au disk electrode. To our best knowledge, this is the first successful application of semi-covalent imprinting for preparation of an MIP for selective protein determination.

The HSA imprinting, and then releasing from the MIP-HSA was confirmed by the DPV, EIS, XPS, and PM-IRRAS measurements as well as by AFM imaging.

The DPV response of the MIP-HSA chemosensor to the HSA concentration in solution was linear in the range of 12 to $300 \mathrm{pM}(0.8$ to $20 \mu \mathrm{g} / \mathrm{mL}) \mathrm{HSA}$ with the limit of HSA detection of $0.25 \mathrm{pM}(16.6 \mathrm{ng} / \mathrm{mL})$. For EIS, it was 60 to $1200 \mathrm{pM}$ (4 to $80 \mu \mathrm{g} / \mathrm{mL})$ and $12 \mathrm{pM}(0.8$ $\mu \mathrm{g} / \mathrm{mL}$ ), respectively. Those ranges are far lower than that afforded by the colorimetric Bromocresol Green protocol (Doumas et al. 1997). Moreover, a very high imprinting factor exceeding 20 strongly proved that semi-covalent imprinting allowed forming a large number of very well defined molecular cavities with high affinity to the HSA molecules.

Selectivity of the MIP-HSA chemosensor against the myoglobin and cytochrome $c$ peptide interferences was excellent, and it was high with respect to the lysozyme protein. Moreover, the chemosensor was not responsive to low-(molecular weight) interferences encountered in the analyzed blood and urine samples.

The MIP-HSA chemosensor is promising for HSA determination in human serum samples.

\section{Acknowledgements}

We thank Dr. Wojciech Lisowski for discussion. We acknowledge the Foundation for Polish Science for supporting fellowship of M.C. (Project MPD/2009/1), the Polish National Science

Center (Grant 2011/01/B/ST5/03796 to K.N.) and the National Science Foundation (Grant 
1401188 to F.D.) for financial support, and for supporting the purchase of the VSP potentiostat/galvanostat of Bio-Logic, SAS (Project NCN 2012/07/D/ST5/02241 to Piotr Pięta).

\section{References}

Bergmann, N.M., Peppas, N.A., 2008. Molecularly imprinted polymers with specific recognition for macromolecules and proteins. Prog. Polym. Sci. 33, 271-288.

Bossi, A., Bonini, F., Turner, A.P.F., Piletsky, S.A., 2007. Molecularly imprinted polymers for the recognition of proteins: The state of the art. Biosens. Bioelectron. 22, 1131-1137.

Caballero, D., Martinez, E., Bausells, J., Errachid, A., Samitier, J., 2012. Impedimetric immunosensor for human serum albumin detection on a direct aldehyde-functionalized silicon nitride surface. Anal. Chim. Acta 720, 43-48.

Curry, S., Mandelkow, H., Brick, P., Franks, N., 1998. Crystal structure of human serum albumin complexed with fatty acid reveals an asymmetric distribution of binding sites. Nat. Struct. Biol. 5, 827-835.

Danesh, J., Collins, R., Appleby, P., Peto, R., 1998. Association of fibrinogen, C-reactive protein, albumin, or leukocyte count with coronary heart disease - Meta-analyses of prospective studies. Jama-J. Am. Med. Assoc. 279, 1477-1482.

Doumas, B.T., Watson, W.A., Biggs, H.G., 1997. Albumin standards and the measurement of serum albumin with Bromcresol Green. Clin. Chim. Acta 258, 21-30.

Erickson, H.P., 2009. Size and shape of protein molecules at the nanometer level determined by sedimentation, gel filtration, and electron microscopy. Biol. Proced. Online 11, 32-51.

Greipp, P.R., San Miguel, J., Durie, B.G.M., Crowley, J.J., Barlogie, B., Blade, J., Boccadoro, M., Child, J.A., Harousseau, J.L., Kyle, R.A., Lahuerta, J.J., Ludwig, H., Morgan, G., Powles, R., Shimizu, K., Shustik, C., Sonneveld, P., Tosi, P., Turesson, I., Westin, J., 2005. International staging system for multiple myeloma. J. Clin. Oncol. 23, 3412-3420.

Hawe, A., Sutter, M., Jiskoot, W., 2008. Extrinsic fluorescent dyes as tools for protein characterization. Pharm. Res. 25, 1487-1499.

Hoogenberg, K., Sluiter, W.J., Dullaart, R.P., 1993. Effect of growth hormone and insulin-like growth factor I on urinary albumin excretion: studies in acromegaly and growth hormone deficiency. Acta endocrinol. 129, 151-157.

Huynh, T.P., Bikram, K.C.C., Lisowski, W., D'Souza, F., Kutner, W., 2013a. Molecularly imprinted polymer of bis(2,2'-bithienyl)methanes for selective determination of adrenaline. Bioelectrochemistry 93, 37-45.

Huynh, T.P., Pietrzyk-Le, A., Bikram, K.C.C., Noworyta, K.R., Sobczak, J.W., Sharma, P.S., D'Souza, F., Kutner, W., 2013c. Electrochemically synthesized molecularly imprinted polymer of thiophene derivatives for flow-injection analysis determination of adenosine-5'-triphosphate (ATP). Biosen. Bioelectron. 41, 634-641.

Huynh, T.P., Sosnowska, M., Sobczak, J.W., Kc, C.B., Nesterov, V.N., D'Souza, F., Kutner, W., 2013d. Simultaneous chronoamperometry and piezoelectric microgravimetry determination of 
nitroaromatic explosives using molecularly imprinted thiophene polymers. Anal. Chem. 85, 8361-8368.

Inoue, Y., Kuwahara, A., Ohmori, K., Sunayama, H., Ooya, T., Takeuchi, T., 2013. Fluorescent molecularly imprinted polymer thin films for specific protein detection prepared with dansyl ethylenediamine-conjugated $O$-acryloyl L-hydroxyproline. Biosens. Bioelectron. 48, 113-119.

Keay, G., Doxey, D.L., 1984. A Study of theiInteraction between Bromocresol Green-dye and bovine, ovine and equine serum globulins. Vet. Res. Commun. 8, 25-32.

Kessler, M.A., Meinitzer, A., Petek, W., Wolfbeis, O.S., 1997. Microalbuminuria and borderlineincreased albumin excretion determined with a centrifugal analyzer and the Albumin Blue 580 fluorescence assay. Clin. Chem. 43, 996-1002.

Klibanov, A.M., 2001. Improving enzymes by using them in organic solvents. Nature 409, 241246.

Kryscio, D.R., Peppas, N.A., 2012. Critical review and perspective of macromolecularly imprinted polymers. Acta Biomater. 8, 461-473.

Li, S.J., Cao, S.S., Whitcombe, M.J., Piletsky, S.A., 2014. Size matters: Challenges in imprinting macromolecules. Prog. Polym. Sci. 39, 145-163.

Lin, T.Y., Hu, C.H., Chou, T.C., 2004. Determination of albumin concentration by MIP-QCM sensor. Biosensors \& bioelectronics 20, 75-81.

Matulis, D., Baumann, C.G., Bloomfield, V.A., Lovrien, R.E., 1999. 1-anilino-8-naphthalene sulfonate as a protein conformational tightening agent. Biopolymers 49, 451-458.

Moreira, F.T.C., Dutra, R.A.F., Noronha, J.P.C., Fernandes, J.C.S., Sales, M.G.F., 2013a. Novel biosensing device for point-of-care applications with plastic antibodies grown on Au-screen printed electrodes. Sens. Actuators, B 182, 733-740.

Moreira, F.T.C., Dutra, R.A.F., Noronha, J.P.C., Sales, M.G.F., 2013b. Electrochemical biosensor based on biomimetic material for myoglobin detection. Electrochim. Acta 107, 481487.

Moreira, F.T.C., Sharma, S., Dutra, R.A.F., Noronha, J.P.C., Cass, A.E.G., Sales, M.G.F., 2013c. Smart plastic antibody material (SPAM) tailored on disposable screen printed electrodes for protein recognition: Application to myoglobin detection. Biosens. Bioelectron. 45, 237-244.

Murch, S.H., Winyard, P.J., Koletzko, S., Wehner, B., Cheema, H.A., Risdon, R.A., Phillips, A.D., Meadows, N., Klein, N.J., Walker-Smith, J.A., 1996. Congenital enterocyte heparan sulphate deficiency with massive albumin loss, secretory diarrhoea, and malnutrition. Lancet 347, 1299-1301.

Peters, J.T., 1995. All About Albumin: Biochemistry, Genetics, and Medical Applications. Academic Press, San Diego, San Diego.

Pietrzyk, A., Suriyanarayanan, S., Kutner, W., Chitta, R., Zandler, M.E., D'Souza, F., 2010a. Molecularly imprinted polymer (MIP) based piezoelectric microgravimetry chemosensor for selective determination of adenine. Biosens. Bioelectron. 25, 2522-2529.

Pietrzyk, A., Suriyanarayanan, S., Kutner, W., Maligaspe, E., Zandler, M.E., D'Souza, F., $2010 b$. Molecularly imprinted poly[bis(2,2'-bithienyl)methane] film with built-in molecular recognition 
sites for a piezoelectric microgravimetry chemosensor for selective determination of dopamine. Bioelectrochemistry 80, 62-72.

Rachkov, A., Minoura, N., 2001. Towards molecularly imprinted polymers selective to peptides and proteins. The epitope approach. Biochim. Biophys. Acta 1544, 255-266.

Rodkey, F.L., 1965. Direct spectrophotometric determination of albumin in human serum. Clin. Chem. 11, 478.

Sellergren, B., 2001. The non-covalent approach to molecular imprinting 23, 113-184.

Sellergren, B., Andersson, L., 1990. Molecular recognition in macroporous polymers prepared by a substrate-analog imprinting strategy. J Org Chem 55, 3381-3383.

Sellergren, B., Lepisto, M., Mosbach, K., 1988. Highly Enantioselective and Substrate-Selective Polymers Obtained by Molecular Imprinting Utilizing Noncovalent Interactions - Nmr and Chromatographic Studies on the Nature of Recognition. J. Am. Chem. Soc. 110, 5853-5860.

Smith, S.E., Williams, J.M., Ando, S., Koide, K., 2014. Time-insensitive fluorescent sensor for human serum albumin and its unusual red shift. Anal. Chem. 86, 2332-2336.

Snozek, C.L.H., Saenger, A.K., Greipp, P.R., Bryant, S.C., Kyle, R.A., Rajkumar, S.V., Katzmann, J.A., 2007. Comparison of bromcresol green and agarose protein electrophoresis for quantitation of serum albumin in multiple myeloma. Clin. Chem. 53, 1099-1103.

Suga, Y., Sunayama, H., Ooya, T., Takeuchi, T., 2013. Molecularly imprinted polymers prepared using protein-conjugated cleavable monomers followed by site-specific post-imprinting introduction of fluorescent reporter molecules. Chem. Commun. 49, 8450-8452.

Sun, Y., Wei, S., Zhao, Y.Y., Hu, X.Y., Fan, J., 2012. Interactions between 4-(2dimethylaminoethyloxy)- $N$-octadecyl-1,8-naphthalimide and serum albumins: Investigation by spectroscopic approach. J. Lumin. 132, 879-886.

Suzuki, Y., Yokoyama, K., 2005. Design and synthesis of intramolecular charge transfer-based fluorescent reagents for the highly-sensitive detection of proteins. J. Am. Chem. Soc. 127, 17799-17802.

Tretjakov, A., Syritski, V., Reut, J., Boroznjak, R., Volobujeva, O., Opik, A., 2013. Surface molecularly imprinted polydopamine films for recognition of immunoglobulin G. Microchim. Acta 180, 1433-1442.

Verheyen, E., Schillemans, J.P., van Wijk, M., Demeniex, M.A., Hennink, W.E., van Nostrum, C.F., 2011. Challenges for the effective molecular imprinting of proteins. Biomaterials 32, 30083020.

Volkova, K.D., Kovalska, V.B., Losytskyy, M.Y., Reis, L.V., Santos, P.F., Almeida, P., Lynch, D.E., Yarmoluk, S.M., 2011. Aza-substituted squaraines for the fluorescent detection of albumins. Dyes Pigm. 90, 41-47.

Webster, D., Bignell, A.H.C., Attwood, E.C., 1974. Assessment of suitability of bromocresol green for determination of serum-albumin. Clin. Chim. Acta 53, 101-108.

Wulff, G., Sarhan, A., 1972. Use of Polymers with Enzyme-Analogous Structures for Resolution of Racemates. Angew Chem Int Edit 11,341-\&. 
Yang, K.G., Zhang, L.H., Liang, Z., Zhang, Y.K., 2012. Protein-imprinted materials: rational design, application and challenges. Anal. Bioanal. Chem. 403, 2173-2183.

Zhang, Z., Long, Y., Nie, L., Yao, S., 2006. Molecularly imprinted thin film self-assembled on piezoelectric quartz crystal surface by the sol-gel process for protein recognition. Biosens. Bioelectron. 21, 1244-1251. 
Fig. 1. (a) The DPV and (b) EIS curves at open circuit potential recorded for ( 1 and 1 ') the bare Au-disk electrode and the MIP film coated electrode in the $0.1 \mathrm{M}$ PBS $(\mathrm{pH}=7.4)$ solution of the $10 \mathrm{mM} \mathrm{K}_{3}\left[\mathrm{Fe}(\mathrm{CN})_{6}\right]$ and $10 \mathrm{mM}$ $\mathrm{K}_{4}\left[\mathrm{Fe}(\mathrm{CN})_{6}\right]$ redox probe (2 and 2 ') before, and then after HSA extraction with $30 \% \mathrm{NaOH}$ at $40{ }^{\circ} \mathrm{C}$ for $(3) 10$ min and (4 and 4') 20 min. Inset in (b) presents the scheme of the modified Randles-Ershler equivalent circuit where $R_{\mathrm{s}}$, $R_{\mathrm{ct}}, C P E$, and $W_{\mathrm{o}}$ stands for the solution resistance, charge transfer resistance, constant phase element, and Warburg impedance, respectively.

Figure 2. The (a) DPV and (b) EIS calibration plots at open circuit potential for human serum albumin, for the ( 1 and $1^{\prime}$ ) MIP and (2 and 2') NIP film coated Au disk electrode in the $0.1 \mathrm{M}$ PBS $(\mathrm{pH}=7.4)$ in solution of the $10 \mathrm{mM}$ $\mathrm{K}_{3}\left[\mathrm{Fe}(\mathrm{CN})_{6}\right]$ and $10 \mathrm{mM} \mathrm{K}_{4}\left[\mathrm{Fe}(\mathrm{CN})_{6}\right]$ redox probe.

Figure 3. The EIS response (charge transfer resistance) at open circuit potential of the MIP-HSA film coated Au disk electrode to (1) human serum albumin, (2) lysozyme, (3) cytochrome $c$, and (4) myoglobin in the $0.1 \mathrm{M}$ PBS $(\mathrm{pH}=7.4)$ solution of the $10 \mathrm{mM} \mathrm{K}_{3}\left[\mathrm{Fe}(\mathrm{CN})_{6}\right]$ and $10 \mathrm{mM} \mathrm{K}_{4}\left[\mathrm{Fe}(\mathrm{CN})_{6}\right]$ redox probe. 
Table 1. The EIS signal (charge transfer resistance) of the MIP-HSA chemosensor to the low-(molecular weight) interferences encountered in human blood or urine.

\begin{tabular}{rlrcc}
\hline $\begin{array}{c}\text { Sample } \\
\text { no. }\end{array}$ & $\begin{array}{c}\text { Analyte or } \\
\text { interference }\end{array}$ & $\begin{array}{c}\text { Molecular } \\
\text { weight, Da }\end{array}$ & Concentration & $\begin{array}{c}\text { The EIS signal (charge } \\
\text { transfer resistance) of the } \\
\text { MIP-HSA chemosensor } \boldsymbol{\Omega}^{\mathrm{a}} \boldsymbol{\Omega}\end{array}$ \\
\hline 1. & HSA & 66400 & 0.82 to $115 \mu \mathrm{g} / \mathrm{mL}$ & 300 to 2900 \\
2. & Creatinine & 113 & $16.5 \mu \mathrm{g} / \mathrm{mL}^{\mathrm{b}, \mathrm{d}}$ & No response \\
3. & Glucose & 180 & $1 \mathrm{mg} / \mathrm{mL}^{\mathrm{c}}$ & 7800 \\
4. & Urea & 60 & $10 \mu \mathrm{mg} / \mathrm{mL}^{\mathrm{b}, \mathrm{d}}$ & No response \\
5. & Uric Acid & 168 & $80 \mu \mathrm{g} / \mathrm{mL}^{\mathrm{c}, \mathrm{d}}$ & No response \\
\hline
\end{tabular}

${ }^{\mathrm{a}}$ Measured at 60 min after analyte or interference addition

${ }^{\mathrm{b}}$ Maximum concentration in urine.

${ }^{\mathrm{c}}$ Maximum concentration in blood.

${ }^{\mathrm{d}}$ The measurement was performed for the highest of the two indicated concentrations. 
Table 2. The EIS signal (charge transfer resistance) of the MIP-HSA electrode to HSA of different concentration in a NORTROL control human serum.

\begin{tabular}{ccccc}
\hline $\begin{array}{c}\text { Sample } \\
\text { no. }\end{array}$ & $\begin{array}{c}\text { The EIS response of the } \\
\text { MIP-HSA electrode } \\
\pm \text { st. dev., } \Omega\end{array}$ & $\begin{array}{c}\text { Amount of HSA } \\
\text { added to NORTROL, } \\
\text { mg/mL }\end{array}$ & $\begin{array}{c}\text { Determined } \\
\text { HSA st. dev., mg/mL }\end{array}$ & $\begin{array}{c}\text { Recovery } \\
\pm \text { st. dev., \% }\end{array}$ \\
\hline 1. & 281 & 0 & - & - \\
2. & $900 \pm 119$ & 24.18 & $23.1 \pm 3.8$ & $96 \pm 15$ \\
3. & $1607 \pm 195$ & 39.90 & $41.1 \pm 6.2$ & $114 \pm 16$ \\
4. & $2134 \pm 209$ & 55.33 & $62.3 \pm 6.6$ & $117 \pm 12$ \\
\hline
\end{tabular}

${ }^{\mathrm{a}}$ Measured at $60 \mathrm{~min}$ after addition of 2,5 $\mu \mathrm{L}$ NORTROL with HSA added. 

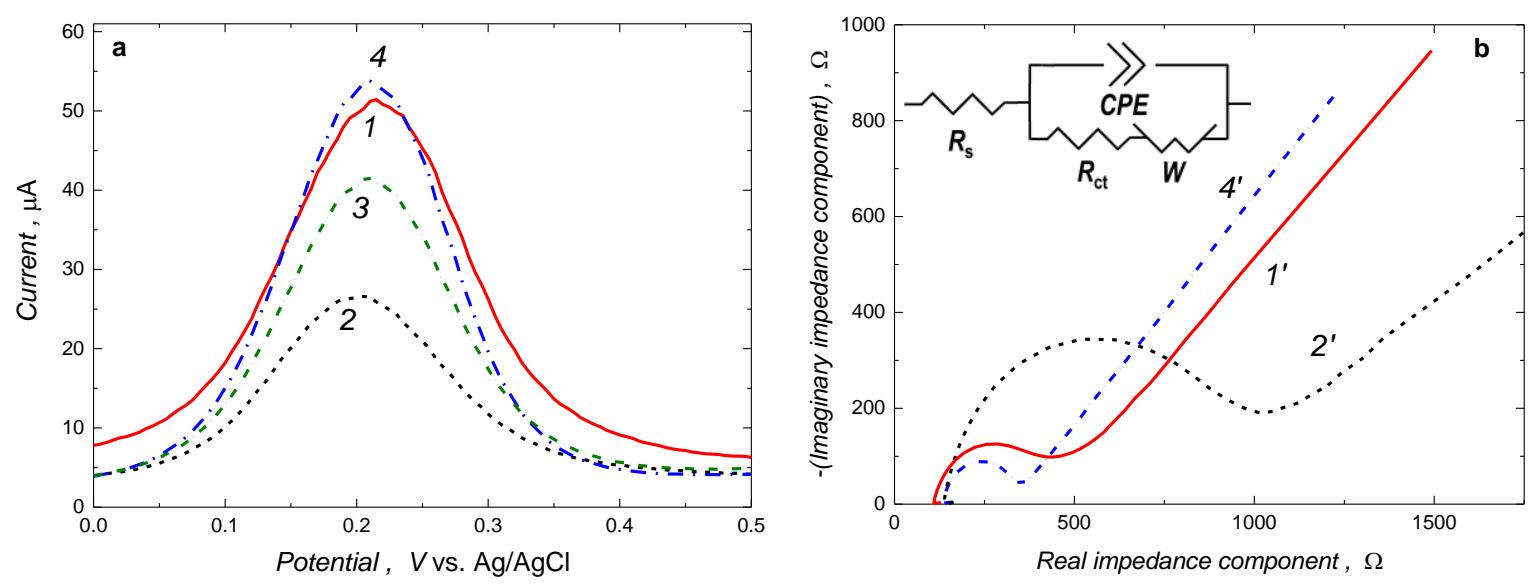

Fig. 1 

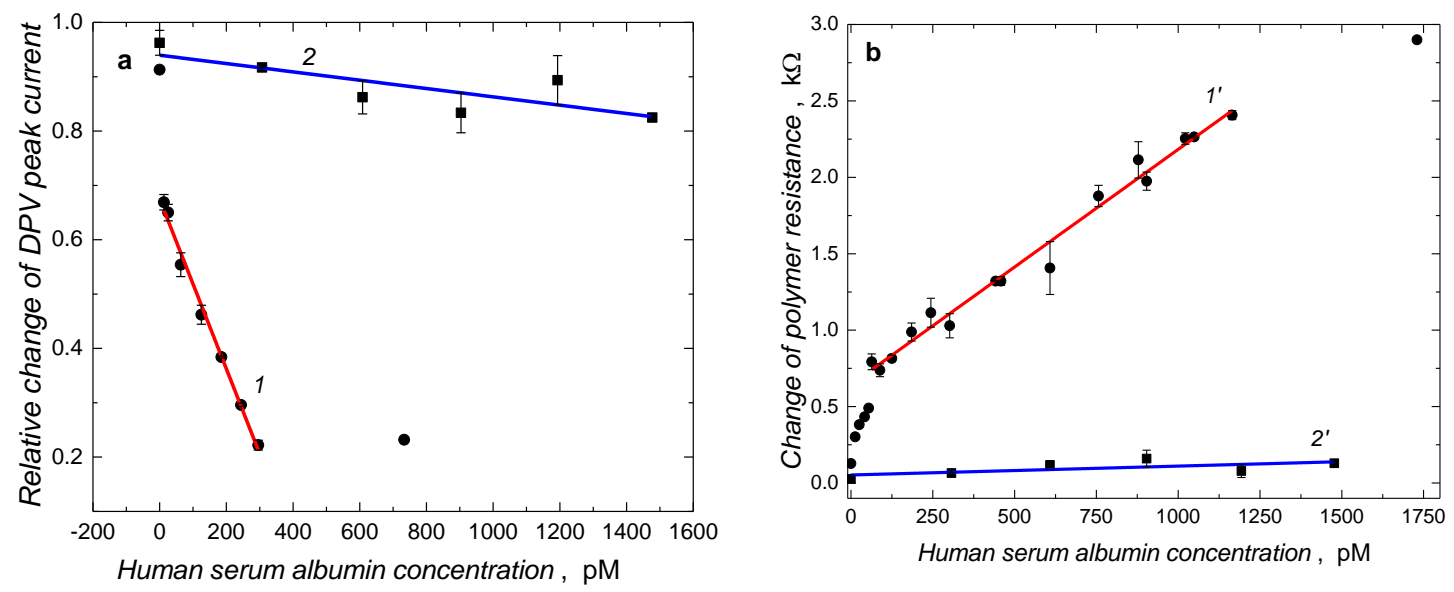

Fig. 2 


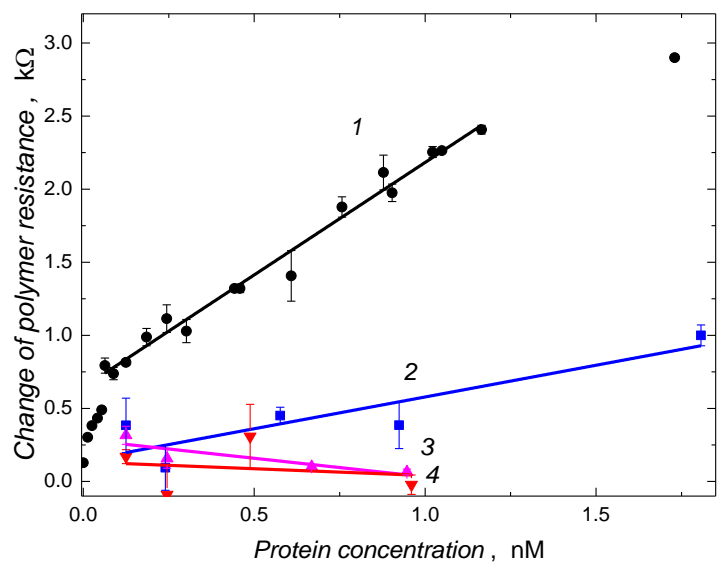

Fig. 3 